\title{
Crescimento diamétrico e tempo de passagem de Minquartia guianensis após manejo na Floresta Nacional do Tapajós
}

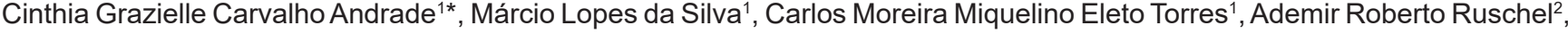 \\ Liniker Fernandes da Silva ${ }^{3}$, Dárlison Fernandes Carvalho de Andrade ${ }^{4}$, Leonardo Pequeno Reis ${ }^{1}$ \\ ${ }^{1}$ Universidade Federal de Viçosa, Campus Universitário, s/n., CEP 36570-000 - Viçosa, MG, Brasil \\ ${ }^{2}$ Embrapa Amazônia Oriental, Trav. Dr. Enéas Pinheiro S/n, CP 48,CEP 66095100, Belém, PA, Brasil \\ ${ }^{3}$ Universidade Federal do Recôncavo da Bahia, Rua Rui Barbosa, 710, Centro, CEP 44380-000, Cruz das Almas, BA, Brasil \\ ${ }^{4}$ Instituto Chico Mendes de Conservação da Biodiversidade, Floresta Nacional de Tapajós, Av. Tapajós, 2267, Laguinho, CEP 68040-000, Santarém, PA, Brasil
}

"Autor correspondente:

grazy_stm@hotmail.com

Termos para indexação:

Produtividade

Amazônia oriental

Corte de seleção

Index terms:

Productivity

Selective felling

Eastern Amazonia

Recebido em 13/02/2017

Aprovado em 30/08/2017

Publicado em 29/09/2017

doi: $10.4336 / 2017 . p f b .37 .91 .1406$
Resumo - Minquartia guianensis Aubl. (acariquara) ocorre nos estados do Acre, Amazonas, Roraima, Pará e Amapá, com grande procura de mercado e utilidade na região Amazônica. Seu principal uso é na construção civil, por apresentar alta durabilidade e reentrâncias em seu tronco, que a tornam de beleza singular e muito valorizada. A maioria das árvores utilizadas apresenta pequenos diâmetros, sendo este um fator crítico para seu manejo e comercialização. Portanto, objetivou-se avaliar o crescimento diamétrico e o ciclo de corte de $M$. guianensis, por meio da análise do tempo de passagem entre as classes diamétricas, no período de 31 anos, após a exploração florestal, na Floresta Nacional do Tapajós, estado do Pará. Foram estabelecidos cinco tratamentos, e em cada um foram instaladas 12 parcelas permanentes de 0,25 ha, nas quais foram medidas todas as árvores com diâmetro a $1,30 \mathrm{~m}$ do solo (DAP) $\geq 5 \mathrm{~cm}$, de 1981 a 2012. As árvores com DAP $\leq 50 \mathrm{~cm}$ têm potencial para serem manejadas, em razão da diminuição do crescimento diamétrico a partir do DAP $\geq 50 \mathrm{~cm}$. Esses resultados poderão servir de base para subsidiar decisões silviculturais no manejo sustentável da espécie.

\section{Diametric growth and time of passage of Minquartia guianensis after logging at Tapajós National Forest, Brazil}

\begin{abstract}
Minquartia guianensis Aubl. (acariquara) occurs at Acre, Amazonas, Roraima, Pará and Amapá states, with a great market demand and utility in the Amazon region. It is used mainly in civil construction due to its high durability and singular beauty, because of reentrances in its trunk, resulting in high market value. Most of used trees present small diameters, what is a critical factor for their management and commercialization. Thus, the objective of this study was to evaluate $M$. guianensis diametric growth and cutting cycle through the analysis of time of passage among diameter classes, 31 years after logging, at Tapajós National Forest, Pará State, Brazil. Five treatments were established and in each one 12 permanent sample plots of 0.25 ha were installed. All trees with diameter at $1.3 \mathrm{~m}$ above ground level (DBH) $\geq 5 \mathrm{~cm}$ were measured from 1981 to 2012 . Trees with $\mathrm{DBH} \leq 50 \mathrm{~cm}$ have potential to be managed, due to the growth stagnation presented in larger diameter classes. These results may support silvicultural decisions to sustainable management.
\end{abstract}




\section{Introdução}

A floresta Amazônica apresenta elevada diversidade de espécies arbóreas potenciais para a produção de madeira. Apesar disso, o sistema de manejo adotado em muitos locais é a exploração convencional, acarretando degradação e extinção de espécies, devido ao uso de práticas predatórias. Dessa forma, é importante que seja implementado o manejo florestal sustentável, que se baseia na capacidade do povoamento florestal produzir no período do ciclo de corte um estoque de madeira compatível ao explorado (Souza et al., 2015).

Para o cálculo correto do rendimento sustentável, são necessárias informações sobre o crescimento das espécies arbóreas, especialmente daquelas de interesse econômico, em áreas exploradas e submetidas a tratamentos silviculturais, para se estabelecer o ciclo de corte e o volume disponível periodicamente para a exploração (Cunha \& Finger, 2013; Taffarel et al., 2014).

$\mathrm{Na}$ Amazônia, estudos voltados ao conhecimento sobre o crescimento, a idade relativa e o ciclo de corte de espécies são úteis para a obtenção da maximização do incremento da floresta de produção. No entanto, para muitas espécies, como Minquartia guianensis Aubl. (Olacaceae), estes estudos ainda são escassos. A espécie, conhecida popularmente como acariquara, acariquara-roxa ou aquariquara, ocorre na América Central, da Nicarágua ao Panamá, e na Bacia Amazônica (Acre, Amazonas, Roraima, Pará e Amapá). A madeira é muito utilizada na indústria madeireira, para a fabricação de postes de uso em redes de energia elétrica, estacas, dormentes e mourões (Camargo \& Ferraz, 2005).

A acariquara apresenta ampla ocorrência e abundância, possuindo alta durabilidade e beleza singular, em razão das reentrâncias em seu tronco, o que a torna uma espécie de grande demanda, principalmente, na construção civil (Camargo \& Ferraz, 2005). Contudo, a maioria dos indivíduos utilizados apresenta diâmetro pequeno, sendo este um fator crítico para seu manejo e comercialização, de acordo com a legislação vigente.

O objetivo desse estudo foi analisar o crescimento e o ciclo de corte de $M$. guianensis, por meio da análise do tempo de passagem entre as classes diamétricas, no período de 31 anos após a exploração florestal, na Floresta Nacional do Tapajós, estado do Pará.

\section{Material e métodos}

\section{Área de estudo}

A pesquisa foi desenvolvida na Floresta Nacional do Tapajós (Flona do Tapajós) no Município de Belterra, PA, ao longo da Rodovia Santarém - Cuiabá à altura do km-114, entre as coordenadas $3^{\circ} 18^{\prime} \mathrm{S}$ a $3^{\circ} 19^{\prime} \mathrm{S}$ e $54^{\circ} 56^{\prime} \mathrm{W}$ a $54^{\circ} 57^{\prime} \mathrm{W}$. O clima da região, segundo a classificação de Köppen, é do tipo Ami (quente e úmido), caracterizado por um período seco de dois a quatro meses, com maior intensidade de chuvas entre os meses de dezembro a junho (Espírito-Santo et al., 2005). A temperatura média anual é de $25,3{ }^{\circ} \mathrm{C}$ e a umidade relativa do ar de 95\% (Instituto Nacional de Meteorologia, 2016).

O solo predominante na área é do tipo álico de Latossolo amarelo moderado, com a presença também de Latossolo amarelo concrecionário, derivado de argilitos (Carvalho, 2002). A área de estudo está localizada na tipologia Floresta Ombrófila Densa, caracterizada pelo domínio de árvores de grande porte (IBGE, 2012).

\section{Coleta de dados}

O estudo foi constituído por cinco tratamentos instalados em 180 ha, dos quais 144 ha são referentes à exploração realizada em 1982 (4 tratamentos) e o quinto ao tratamento controle, com 36 ha, instalado em 1983, em floresta primária não explorada. Conforme detalhado em Oliveira et al. (2006): $\mathrm{T}_{0}$ : tratamento controle, floresta sem intervenção; $T_{1}$ : Exploração de espécies comerciais madeireiras com diâmetro a $1,30 \mathrm{~m}$ do solo (DAP) $\geq$ $45 \mathrm{~cm}$, sem nenhuma intervenção posterior; $\mathrm{T}_{2}, \mathrm{~T}_{3} \mathrm{e} \mathrm{T}_{4}$ : Exploração de árvores comerciais com DAP $\geq 55 \mathrm{~cm}+$ desbaste de espécies não comerciais com DAP a partir de $45 \mathrm{~cm}$, para reduzir a área basal em $20 \%, 30 \%$ e $50 \%$ da original, respectivamente.

Foram instaladas, ao acaso, 12 parcelas de 0,25 ha por tratamento, totalizando 60 parcelas. Nestas, foram medidas, periodicamente, as árvores com DAP $\geq 5 \mathrm{~cm}$.

A metodologia para instalação e medição das parcelas seguiu as definições de Silva et al. (2005). Os dados foram coletados em oito ocasiões: a primeira medição em 1981 (antes da exploração), exceto o tratamento controle, que teve sua primeira medição em 1983. As remedições 
após a exploração florestal foram em 1983, 1985, 1987, 1989, 1995, 2008 e 2012. A aplicação dos tratamentos silviculturais foi iniciada em 1993 e concluída em 1994, doze anos após a exploração florestal.

A área experimental em 1997, após um intenso período de estiagem gerado pelo El Ninõ, foi atingida por um incêndio acidental que afetou 19 parcelas permanentes $\left(\mathrm{T}_{0}=6 ; \mathrm{T}_{1}=2 ; \mathrm{T}_{2}=5 ; \mathrm{T}_{3}=0 ; \mathrm{T}_{4}=6\right)$ (Oliveira et al., 2006). Neste trabalho foram utilizadas as 60 parcelas, ou seja, não foram excluídas as que foram atingidas pelo fogo.

\section{Incremento diamétrico e tempo de passagem entre classes diamétricas}

Foram utilizados os dados das medições periódicas de todas as árvores com DAP $\geq 5 \mathrm{~cm}$ para calcular, separadamente, o incremento periódico anual médio em diâmetro de cada indivíduo arbóreo e para estimar a idade das árvores de Minquartia guianensis na área de estudo.

Além disso, foi calculada a relação incremento periódico anual médio (IPA) e o tempo médio de passagem por classe diamétrica $\left(\mathrm{T}_{0}=5-50 \mathrm{~cm} ; \mathrm{T}_{1}\right.$, $\mathrm{T}_{2}, \mathrm{~T}_{3}$ e $\mathrm{T}_{4}: 5-75 \mathrm{~cm}$ ). O IPA médio das árvores de M. guianensis foi determinado pela razão do tempo e a diferença dos diâmetros medidos em dois intervalos de tempo.

Os resultados de IPA das árvores de M. guianensis e os seus respectivos valores de diâmetro, foram relacionados em um modelo de regressão linear (Equação 1), conforme proposto por Souza et al. (1993).

$$
I P A_{i}=\beta_{0}+\beta_{l} D_{i}+\beta_{l} D_{i}^{2}+e_{i}
$$

em que: $I P A_{i}=$ estimador do incremento periódico anual médio em diâmetro $\left(\mathrm{cm} \mathrm{ano}^{-1}\right)$ da i-ésima classe de diâmetro (cm); $\beta_{0}, \beta_{1}$ e $\beta_{2}=$ coeficientes da regressão; e $D_{i}=$ diâmetro a 1,30 $\mathrm{m}$ do solo $(\mathrm{cm})$ para $\mathrm{i}=1,2,3, \ldots, \mathrm{n}$-ésima classe de diâmetro; $e_{i}=$ erro aleatório.

Após o ajuste, estimou-se o incremento diamétrico para o valor da menor classe de diâmetro $(5 \mathrm{~cm})$. Em seguida, foi determinado o número de anos necessários para que as árvores do estoque em regeneração ingressassem na classe de diâmetro mínimo mensurável $\left(D_{1}\right)$. A soma do diâmetro $\left(D_{1}\right)$, juntamente com seu respectivo incremento (IPA $A_{\mathrm{i}}$ ), foi o próximo valor da variável independente $\left(\mathrm{D}_{2}\right)$ a ser substituída na equação. Assim, foram estabelecidos sucessivos valores de diâmetro e incremento de acordo com as idades relativas em anos (Souza \& Soares, 2013).

O tempo de passagem entre as classes diamétricas, com amplitude de $10 \mathrm{~cm}$, foi obtido por meio da diferença de idades dos DAPs referentes aos limites inferiores de duas classes diamétricas sucessivas, que foi utilizada para estimar o número de anos para que $M$. guianensis alcançasse seu diâmetro de corte, definindo a idade mínima e máxima por classe diamétrica em cada tratamento, conforme metodologia estabelecida por Souza et al. (1993).

\section{Resultados e discussão}

Em todos os tratamentos avaliados na classe de 5-45 $\mathrm{cm}$ ocorreu maior número de árvores amostradas, com destaque para $\mathrm{T}_{2}$ e $\mathrm{T}_{3}$ com maior e menor valor em todos os anos, respectivamente. Além disso, de 1981 para 2012 houve uma redução de $8\left(\mathrm{~T}_{1}\right), 9\left(\mathrm{~T}_{2}\right), 8\left(\mathrm{~T}_{3}\right)$ e $8\left(\mathrm{~T}_{4}\right)$ árvores avaliadas, devido `a mortalidade (Tabela 1). Por outro lado, $\mathrm{T}_{0}$ que teve sua primeira medição em 1983, apresentou comportamento diferente com acréscimo de 4 árvores até o ano de 2012. Em todos os tratamentos foi verificada a mesma tendência quando avaliadas as demais classes diamétricas.

A estatística descritiva (média, mediana, valor mínimo e máximo e desvio padrão) do número de árvores por ha dos tratamentos avaliados no decorrer dos anos, indicou uma alta variabilidade encontrada nas parcelas quanto à ocorrência da espécie (abundância) (Tabela 2).

Nos tratamentos $T_{1}, T_{2}$ e $T_{3}$ houve aumento do incremento diamétrico entre 1981 e 1987, até o final do terceiro ano após manejo, provável pelo efeito da abertura do dossel causada pela exploração florestal, que pode ter contribuído para o crescimento das árvores. No entanto, no $\mathrm{T}_{4}$ ocorreu decréscimo no crescimento durante o período de 1983 a 1987. A partir de 1987 houve redução do incremento em todos os tratamentos, exceto no $\mathrm{T}_{1}$.

Reis et al. (2014), estudando o IPA de Parkia gigantocarpa Ducke plantadas em clareiras, no Município de Paragominas, PA, concluíram que o incremento periódico anual (IPA) foi elevado nos primeiros anos pós-colheita, tendo uma redução no decorrer dos anos, devido ao fechamento do dossel da floresta. Silva et al. (1995) observaram a mesma tendência, redução do incremento diamétrico após seis anos de exploração para toda a comunidade florestal. 
Tabela 1. Número de árvores amostradas por classe diamétrica e ano de medição nos tratamentos avaliados na Floresta Nacional do Tapajós, Belterra, PA.

\begin{tabular}{|c|c|c|c|c|c|c|c|c|c|c|c|c|c|c|}
\hline Ano & DAP (cm) & $\mathbf{T}_{0}$ & $T_{1}$ & $T_{2}$ & $\mathbf{T}_{3}$ & $T_{4}$ & Total & Ano & $\mathbf{T}_{0}$ & $T_{1}$ & $\mathbf{T}_{2}$ & $\mathbf{T}_{3}$ & $\mathbf{T}_{4}$ & Total \\
\hline \multirow{8}{*}{1981} & $5-15$ & - & 13 & 12 & 7 & 12 & 44 & \multirow{8}{*}{1995} & 21 & 8 & 14 & 5 & 11 & 59 \\
\hline & $15-25$ & - & 4 & 14 & 9 & 8 & 35 & & 4 & 5 & 8 & 3 & 11 & 31 \\
\hline & $25-35$ & - & 7 & 12 & 4 & 7 & 30 & & 2 & 2 & 10 & 4 & 2 & 20 \\
\hline & $35-45$ & - & 0 & 8 & 2 & 5 & 15 & & 2 & 3 & 10 & 1 & 5 & 21 \\
\hline & $45-55$ & - & 2 & 3 & 3 & 1 & 9 & & 2 & 1 & 4 & 2 & 3 & 12 \\
\hline & $55-65$ & - & 3 & 4 & 2 & 2 & 11 & & --- & 3 & 4 & 2 & 1 & 10 \\
\hline & $>65$ & - & 3 & 4 & 1 & 1 & 9 & & --- & 4 & 5 & 2 & 2 & 13 \\
\hline & Total & - & 32 & 57 & 28 & 36 & 153 & & 31 & 26 & 55 & 19 & 35 & 166 \\
\hline \multirow{8}{*}{1983} & $5-15$ & 17 & 8 & 13 & 5 & 11 & 54 & \multirow{8}{*}{2008} & 20 & 10 & 9 & 6 & 6 & 51 \\
\hline & $15-25$ & 6 & 5 & 12 & 9 & 9 & 41 & & 3 & 2 & 7 & 3 & 7 & 22 \\
\hline & $25-35$ & 0 & 4 & 10 & 2 & 5 & 21 & & 3 & 1 & 8 & 4 & 4 & 20 \\
\hline & $35-45$ & 2 & 1 & 8 & 1 & 3 & 15 & & 2 & 4 & 8 & 0 & 3 & 17 \\
\hline & $45-55$ & 2 & 2 & 4 & 3 & 3 & 14 & & 2 & 0 & 7 & 3 & 2 & 14 \\
\hline & $55-65$ & --- & 3 & 4 & 1 & 2 & 10 & & --- & 2 & 3 & 2 & 3 & 10 \\
\hline & $>65$ & --- & 3 & 4 & 2 & 1 & 10 & & --- & 5 & 6 & 2 & 1 & 14 \\
\hline & Total & 27 & 26 & 55 & 23 & 34 & 165 & & 30 & 24 & 48 & 20 & 26 & 148 \\
\hline \multirow{8}{*}{1987} & $5-15$ & 19 & 9 & 14 & 4 & 12 & 58 & \multirow{8}{*}{2012} & 19 & 10 & 10 & 6 & 8 & 53 \\
\hline & $15-25$ & 4 & 5 & 12 & 6 & 9 & 36 & & 5 & 2 & 5 & 3 & 6 & 21 \\
\hline & $25-35$ & 2 & 3 & 9 & 4 & 5 & 23 & & 2 & 1 & 9 & 2 & 5 & 19 \\
\hline & $35-45$ & 2 & 2 & 10 & 1 & 3 & 18 & & 2 & 3 & 7 & 2 & 3 & 17 \\
\hline & $45-55$ & 2 & 1 & 3 & 3 & 3 & 12 & & 3 & 1 & 8 & 1 & 2 & 15 \\
\hline & $55-65$ & --- & 3 & 5 & 1 & 2 & 11 & & --- & 2 & 3 & 4 & 3 & 12 \\
\hline & $>65$ & --- & 4 & 4 & 2 & 1 & 11 & & --- & 5 & 6 & 2 & 1 & 14 \\
\hline & Total & 29 & 27 & 57 & 21 & 35 & 169 & & 31 & 24 & 48 & 20 & 28 & 151 \\
\hline \multirow{8}{*}{1989} & $5-15$ & 17 & 10 & 13 & 4 & 12 & 56 & & & & & & & \\
\hline & $15-25$ & 4 & 5 & 12 & 4 & 9 & 34 & & & & & & & \\
\hline & $25-35$ & 2 & 2 & 8 & 4 & 5 & 21 & & & & & & & \\
\hline & $35-45$ & 2 & 3 & 11 & 1 & 3 & 20 & & & & & & & \\
\hline & $45-55$ & 2 & 1 & 3 & 3 & 3 & 12 & & & & & & & \\
\hline & $55-65$ & 0 & 3 & 4 & 1 & 2 & 10 & & & & & & & \\
\hline & $>65$ & 0 & 4 & 5 & 2 & 1 & 12 & & & & & & & \\
\hline & Total & 27 & 28 & 56 & 19 & 35 & 165 & & & & & & & \\
\hline
\end{tabular}

$\mathrm{T}_{0}$ : tratamento controle, floresta sem intervenção; $\mathrm{T}_{1}$ : exploração de espécies comerciais madeireiras com diâmetro a $1,30 \mathrm{~m}$ do solo (DAP) $\geq 45$ $\mathrm{cm}$, sem nenhuma intervenção posterior; $\mathrm{T}_{2} \mathrm{~T}_{3} \mathrm{eT}_{4}$ : exploração de árvores comerciais com $\mathrm{DAP} \geq 55 \mathrm{~cm}+$ desbaste de espécies não comerciais com DAP a partir de $45 \mathrm{~cm}$, para reduzir a área basal em $20 \%, 30 \%$ e $50 \%$ da original, respectivamente. * No tratamento $\mathrm{T}_{0}$ não houve medição em 1981.

Os tratos silviculturais aplicados em 1994 nos tratamentos $\mathrm{T}_{2}, \mathrm{~T}_{3}$ e $\mathrm{T}_{4}$ promoveram maior crescimento no $\mathrm{T}_{4}$ entre 1995-2008, possivelmente, devido à abertura do dossel proporcionada pela maior intensidade de redução da área basal (50\%). O aumento do incremento entre 1995 e 2008 em todos os tratamentos, com exceção do $\mathrm{T}_{2}$, pode ter relação com o incêndio acidental ocorrido em 1997 na área de estudo. Apesar de ter atingido aproximadamente um terço $(19 / 60)$ das parcelas permanentes instaladas, o evento favoreceu a abertura de clareiras, que pode ter contribuído para o crescimento das árvores. Além disso, o incêndio não interferiu de maneira intensa na dinâmica populacional da espécie. Quando se analisa a mortalidade das árvores, apenas 3 parcelas das 19 atingidas pelo fogo apresentaram indivíduos mortos de Minquartia guianensis $\left(2 \mathrm{em} \mathrm{T}_{0}\right.$ e 2 em $\mathrm{T}_{2}$ ). 
Tabela 2. Estatística descritiva dos dados de densidade de árvores $\left(\operatorname{arv~ha}^{-1}\right)$ monitoradas, pelo período de 31 anos, em 60 parcelas de 50 m x 50 m, instaladas na Floresta Nacional do Tapajós, Belterra, PA.

\begin{tabular}{|c|c|c|c|c|c|c|c|}
\hline Tratamentos & Ano & Média & Mediana & $\begin{array}{l}\text { Desvio } \\
\text { padrão }\end{array}$ & Mínimo & Máximo & $\begin{array}{c}\text { Margem de erro } \\
(95 \%)\end{array}$ \\
\hline T0 & 1981 & $\begin{array}{ll}--- \\
-\cdots\end{array}$ & ---- & $\begin{array}{ll}--- \\
---\end{array}$ & $\begin{array}{ll}--- \\
---\end{array}$ & ---- & $\begin{array}{ll}--- \\
--\end{array}$ \\
\hline T0 & 1983 & 9,0 & 6 & 9,67 & 0 & 28 & 6,14 \\
\hline T0 & 1987 & 9,7 & 6 & 9,41 & 0 & 28 & 5,98 \\
\hline T0 & 1989 & 9,0 & 4 & 9,05 & 0 & 28 & 5,75 \\
\hline T0 & 1995 & 10,3 & 8 & 8,44 & 0 & 28 & 5,36 \\
\hline T0 & 2008 & 10,0 & 8 & 6,71 & 4 & 24 & 4,27 \\
\hline T0 & 2012 & 10,3 & 8 & 6,49 & 4 & 20 & 4,12 \\
\hline $\mathrm{T} 1$ & 1981 & 10,7 & 10 & 3,94 & 4 & 16 & 2,50 \\
\hline $\mathrm{T} 1$ & 1983 & 9,0 & 8 & 5,15 & 0 & 16 & 3,27 \\
\hline $\mathrm{T} 1$ & 1987 & 9,0 & 8 & 5,15 & 0 & 16 & 3,27 \\
\hline $\mathrm{T} 1$ & 1989 & 9,3 & 8 & 5,48 & 0 & 16 & 3,48 \\
\hline $\mathrm{T} 1$ & 1995 & 9,0 & 8 & 5,94 & 0 & 16 & 3,77 \\
\hline $\mathrm{T} 1$ & 2008 & 8,0 & 8 & 5,12 & 0 & 16 & 3,25 \\
\hline $\mathrm{T} 1$ & 2012 & 8,0 & 8 & 4,51 & 0 & 16 & 2,87 \\
\hline $\mathrm{T} 2$ & 1981 & 19,0 & 22 & 12,55 & 0 & 44 & 7,97 \\
\hline $\mathrm{T} 2$ & 1983 & 18,3 & 20 & 12,82 & 0 & 44 & 8,14 \\
\hline $\mathrm{T} 2$ & 1987 & 19,0 & 20 & 12,78 & 0 & 44 & 8,12 \\
\hline $\mathrm{T} 2$ & 1989 & 18,7 & 20 & 13,68 & 0 & 44 & 8,69 \\
\hline $\mathrm{T} 2$ & 1995 & 18,3 & 20 & 12,93 & 0 & 40 & 8,21 \\
\hline $\mathrm{T} 2$ & 2008 & 16,0 & 18 & 11,57 & 0 & 40 & 7,35 \\
\hline $\mathrm{T} 2$ & 2012 & 16,0 & 18 & 11,57 & 0 & 40 & 7,35 \\
\hline $\mathrm{T} 3$ & 1981 & 9,3 & 12 & 5,99 & 0 & 16 & 3,80 \\
\hline $\mathrm{T} 3$ & 1983 & 7,7 & 8 & 5,25 & 0 & 16 & 3,33 \\
\hline $\mathrm{T} 3$ & 1987 & 7,0 & 8 & 4,22 & 0 & 16 & 2,68 \\
\hline $\mathrm{T} 3$ & 1989 & 6,3 & 8 & 4,33 & 0 & 16 & 2,75 \\
\hline $\mathrm{T} 3$ & 1995 & 6,3 & 6 & 4,66 & 0 & 16 & 2,96 \\
\hline T3 & 2008 & 6,7 & 6 & 5,21 & 0 & 16 & 3,31 \\
\hline $\mathrm{T} 3$ & 2012 & 6,7 & 6 & 5,21 & 0 & 16 & 3,31 \\
\hline $\mathrm{T} 4$ & 1981 & 12,0 & 14 & 5,66 & 4 & 20 & 3,59 \\
\hline $\mathrm{T} 4$ & 1983 & 11,3 & 12 & 5,07 & 4 & 20 & 3,22 \\
\hline $\mathrm{T} 4$ & 1987 & 11,6 & 12 & 5,51 & 4 & 20 & 3,50 \\
\hline $\mathrm{T} 4$ & 1989 & 11,6 & 12 & 5,24 & 4 & 20 & 3,33 \\
\hline $\mathrm{T} 4$ & 1995 & 11,6 & 12 & 6,49 & 4 & 24 & 4,12 \\
\hline $\mathrm{T} 4$ & 2008 & 8,7 & 8 & 3,34 & 4 & 16 & 2,12 \\
\hline $\mathrm{T} 4$ & 2012 & 9,3 & 8 & 3,55 & 4 & 16 & 2,26 \\
\hline
\end{tabular}

Entre 2008 e 2012 ocorreu decréscimo nas taxas de IPA de Minquartia guianensis em todos os tratamentos avaliados, possivelmente devido ao fechamento do dossel florestal pelas espécies emergentes, o que diminuiu a entrada de luz e o incremento em diâmetro no decorrer dos anos até atingir o nível do $\mathrm{T}_{0}$, floresta não explorada, também observado por Costa et al. (2008).

$\mathrm{O}$ incremento periódico anual médio verificado nos tratamentos para árvores com centro de classe de 5 a $75 \mathrm{~cm}$ foi: $\mathrm{T}_{0}\left(0,14 \mathrm{~cm} \mathrm{ano}^{-1}\right), \mathrm{T}_{1}\left(0,21 \mathrm{~cm} \mathrm{ano}^{-1}\right), \mathrm{T}_{2}\left(0,24 \mathrm{~cm} \mathrm{ano}^{-1}\right)$, $\mathrm{T}_{3}\left(0,23 \mathrm{~cm} \mathrm{ano}^{-1}\right) \mathrm{e}_{4}\left(0,22 \mathrm{~cm} \mathrm{ano}^{-1}\right)$. Tanto o incremento observado quanto o estimado obtiveram tendência 
a decrescer à medida que as classes passavam de 50 $\mathrm{cm}$, demonstrando o comportamento de Minquartia guinanesis em classes maiores (Figura 1). O incremento em diâmetro apresentou um ritmo de crescimento semelhante ao encontrado no estudo de Braz et al. (2015).
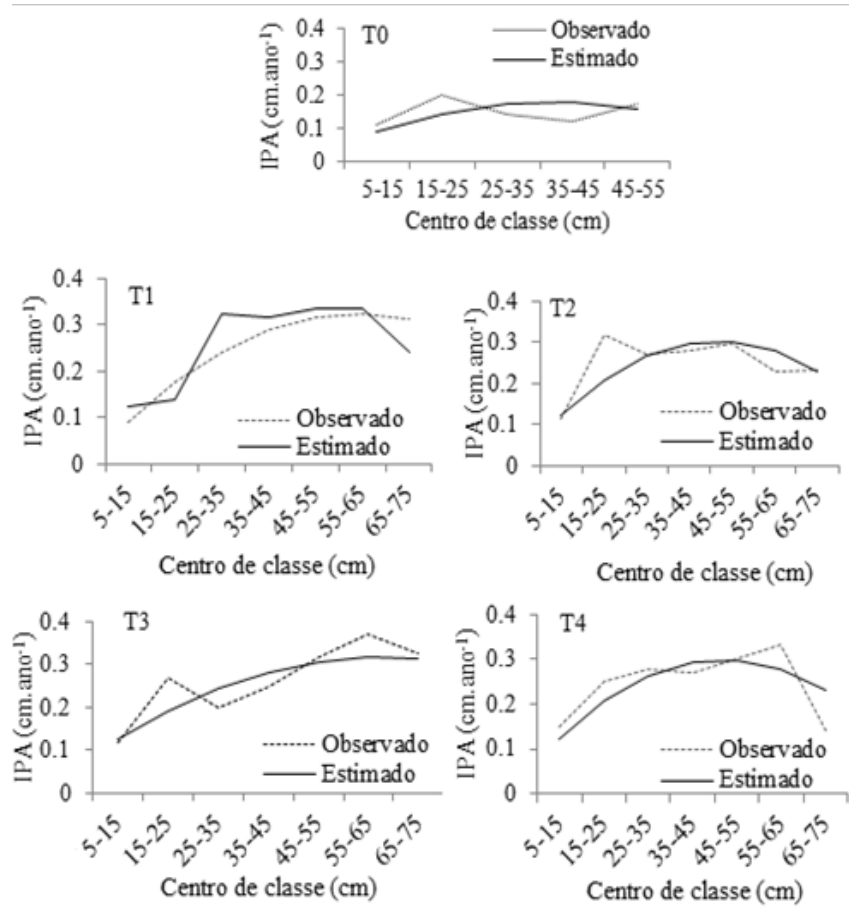

Figura 1. Valores do incremento periódico anual médio (IPA) observados e estimados por classe de diâmetro (DAP) de Minquartia guianensis, na Floresta Nacional do Tapajós, Belterra, PA. $\mathrm{T}_{0}$ : tratamento controle, floresta sem intervenção; $\mathrm{T}_{1}$ : exploração de espécies comerciais madeireiras com diâmetro a $1,30 \mathrm{~m}$ do solo (DAP) $\geq 45 \mathrm{~cm}$, sem nenhuma intervenção posterior; $T_{2}, T_{3}$ e $T_{4}$ : exploração de árvores comerciais com DAP $\geq 55 \mathrm{~cm}+$ desbaste de espécies não comerciais com DAP a partir de $45 \mathrm{~cm}$, para reduzir a área basal em $20 \%, 30 \%$ e $50 \%$ da original, respectivamente.

$\mathrm{O}$ tratamento $\mathrm{T}_{0}$ apresentou menor incremento diamétrico em comparação com os demais. Nos tratamentos em que ocorreu a abertura do dossel pela exploração ou a combinação de exploração florestal com tratamentos silviculturais $\left(\mathrm{T}_{1}, \mathrm{~T}_{2}, \mathrm{~T}_{3}\right.$ e $\left.\mathrm{T}_{4}\right)$, a incidência de luminosidade e a redução da competição por nutrientes e água pode ter contribuído para o aumento do crescimento e da dinâmica das árvores na floresta, conforme apresentado em outros estudos (Oliveira et al., 2006; Azevedo et al., 2012; Cardoso Junior et al., 2014; Taffarel et al., 2014).

Costa et al. (2008) constataram que a exploração florestal, realizada em uma área de terra firme na Floresta Nacional do Tapajós, favoreceu o crescimento das árvores, que no decorrer dos anos foi reduzido, sugerindo a aplicação de tratamentos silviculturais para aumentar $\mathrm{o}$ incremento.

Além disso, apesar de M. guianensis ser tolerante à sombra, apresenta sinais de se beneficiar após distúrbios intermediários (extração de madeira e fogo) causadores de aberturas do dossel, sejam naturais ou resultantes da exploração florestal, nas fases iniciais de desenvolvimento (Silva Jardim \& Soares, 2010; Hirai et al., 2012). Soares et al. (2010) avaliaram o crescimento diamétrico de Sterculia pruriensis (Aubl.) Schum (Axixá) após a exploração florestal e constataram que espécies tolerantes à sombra, apesar de crescerem em condições de dossel fechado e sub-bosque, são favorecidas pela luminosidade oriunda de clareiras, conhecidos por sunflecks, que é a radiação direta que atravessa o dossel e alcança o piso florestal (Marenco \& Vieira, 2005).

Contudo, M. guianensis apresentou crescimento lento em todos os tratamentos, característica comum ao grupo ecológico ao qual pertence. Resultados semelhantes foram encontrados por Azevedo et al. (2008), analisando o efeito de diferentes níveis de exploração e tratamentos silviculturais na formação de agrupamentos ecológico de espécies, que obtiveram IPA de $0,25 \mathrm{~cm}^{a n o}{ }^{-1}$ para M. guianensis, crescimento considerado baixo.

A equação ajustada para os tratamentos avaliados apresentou baixa precisão, pois os valores do coeficiente de determinação ajustado $\left(\overline{\mathrm{R}}^{2}\right)$ foram menores de $20 \%$. $\mathrm{O} \mathrm{T}_{1}$ apresentou maior precisão da estimativa para a relação entre o IPA e o DAP $\left(\overline{\mathrm{R}}^{2}=19,36 \%\right)$ (Tabela 3$)$. 
Tabela 3. Parâmetros estimados pela equação de regressão para a Minquartia guianensis, de acordo com os tratamentos avaliados, na Floresta Nacional do Tapajós, Belterra, PA.

\begin{tabular}{|c|c|c|c|c|c|c|c|c|}
\hline Trat. & $\hat{\boldsymbol{\beta}}_{0}$ & $\hat{\beta}_{1}$ & $\hat{\beta}_{2}$ & $\overline{\mathrm{R}^{2}}(\%)$ & $\overline{\mathrm{R}^{2}}(\%)$ & $\begin{array}{c}\mathrm{S}_{\mathrm{x}, \mathrm{y}} \\
\left(\mathrm{cm} \text { ano }^{-1}\right)\end{array}$ & $\mathbf{F}$ & $\mathbf{N}$ \\
\hline $\mathrm{T}_{0}$ & $\begin{array}{l}0,0556 \\
(0,06)\end{array}$ & $\begin{array}{c}-0,00012 \\
(0,04)\end{array}$ & $\begin{array}{c}0,00782 \\
(0,015)\end{array}$ & 5,89 & 4,49 & 0,117 & 4,19 & 137 \\
\hline $\mathrm{T}_{1}$ & $\begin{array}{c}0,03817 \\
(0,24)\end{array}$ & $\begin{array}{c}-0,00009 \\
(<0,01)\end{array}$ & $\begin{array}{l}0,01056 \\
(<0,01)\end{array}$ & 20,40 & 19,36 & 0,169 & 19,60 & 156 \\
\hline $\mathrm{T}_{2}$ & $\begin{array}{c}0,07334 \\
(0,04)\end{array}$ & $\begin{array}{c}-0,00013 \\
(<0,01)\end{array}$ & $\begin{array}{l}0,01103 \\
(<0,01)\end{array}$ & 7,36 & 6,77 & 0,194 & 12,55 & 319 \\
\hline $\mathrm{T}_{3}$ & $\begin{array}{c}0,08646 \\
(0,11)\end{array}$ & $\begin{array}{c}-0,00007 \\
(0,16)\end{array}$ & $\begin{array}{c}0,00791 \\
(0,03)\end{array}$ & 9,48 & 8,05 & 0,201 & 6,40 & 130 \\
\hline $\mathrm{T}_{4}$ & $\begin{array}{c}0,07126 \\
(0,10)\end{array}$ & $\begin{array}{c}-0,00013 \\
(0,05)\end{array}$ & $\begin{array}{l}0,01095 \\
(<0,01)\end{array}$ & 7,68 & 6,70 & 0,199 & 7,82 & 191 \\
\hline
\end{tabular}

F: significância do modelo; N: número de árvores utilizadas na regressão. Os resultados em parênteses representam os valores do p-valor. T0: tratamento controle, floresta sem intervenção; T1: exploração de espécies comerciais madeireiras com diâmetro a 1,30 m do solo (DAP) $\geq 45 \mathrm{~cm}$, sem nenhuma intervenção posterior; T2, T3 e T4: exploração de árvores comerciais com $\mathrm{DAP} \geq 55 \mathrm{~cm}+$ desbaste de espécies não comerciais com DAP a partir de $45 \mathrm{~cm}$, para reduzir a área basal em $20 \%, 30 \%$ e $50 \%$ da original, respectivamente.

Em todos os tratamentos avaliados, as árvores de $M$. guianensis apresentaram maior densidade nas menores classes de diâmetro (DAP $<50 \mathrm{~cm}$ ), destacando o potencial produtivo da espécie nas classes de 25 a 45 $\mathrm{cm}$ de diâmetro, que são as de interesse do mercado consumidor local. Além disso, o diâmetro máximo observado não ultrapassou $75 \mathrm{~cm}$ e a diminuição da taxa de crescimento ocorreu a partir de $50 \mathrm{~cm}$ (Figura 2).

Em 2012, última medição, apenas os tratamento $\mathrm{T}_{1}$ e $\mathrm{T}_{2}$ apresentaram árvores na classe de $75-95 \mathrm{~cm}$, e o maior diâmetro encontrado foi de $94,6 \mathrm{~cm}\left(\mathrm{~T}_{1}\right)$ e $77 \mathrm{~cm}$ $\left(\mathrm{T}_{2}\right)$ (Figura 3). Braz et al. (2012) afirmam que a maioria das espécies tem reduzido incremento e sobrevivência nas classes maiores, a partir de $70 \mathrm{~cm}$ de diâmetro. No estudo de Nebel (2000) M. guianensis apresentou 234 anos de idade para alcançar $70 \mathrm{~cm}$, sendo que a classe de $30 \mathrm{~cm}$ obteve maior diâmetro médio, indicando que os indivíduos de maior idade podem estar em fase de senescência.

$\mathrm{O} \mathrm{T}_{0}$ apresentou tempo de passagem mais longo (59 anos) para alcançar a classe de $50 \mathrm{~cm}$. Em compensação, $\mathrm{T}_{1}$ apresentou menor tempo (32 anos). Constatou-se que nos tratamentos $\mathrm{T}_{0}$ e $\mathrm{T}_{1}$ uma árvore do estoque de regeneração levará, em média, 55 a 56 anos para ingressar na primeira classe de diâmetro (classe de 5-15 cm), sendo que nos tratamentos $\mathrm{T}_{2}, \mathrm{~T}_{3}$ e $\mathrm{T}_{4} \mathrm{o}$ tempo estimado para o ingresso foi de 40, 40 e 41 anos, respectivamente (Tabela 4).

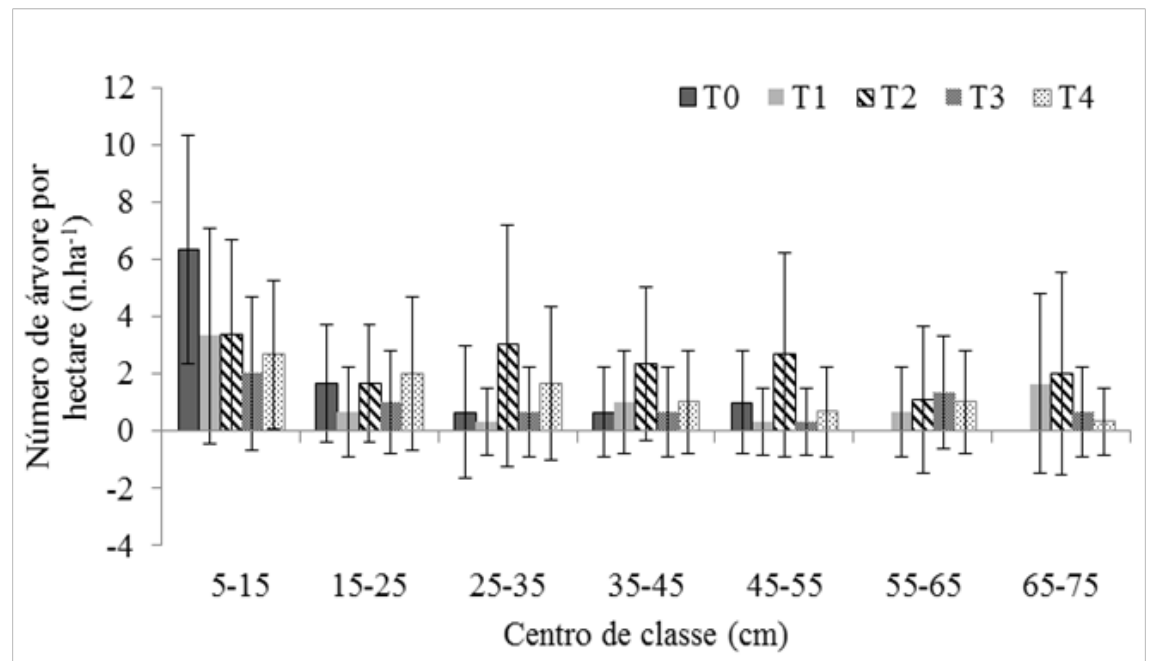

Figura 2. Distribuição do número de árvores por hectare $\left(\mathrm{n} \mathrm{ha}^{-1}\right)$ e classe de diâmetro (DAP) de Minquartia guianensis, com o respectivo desvio padrão, em 2012 na Floresta Nacional do Tapajós, Belterra, PA. $T_{0}$ : tratamento controle, floresta sem intervenção; $T_{1}$ : exploração de espécies comerciais madeireiras com diâmetro a $1,30 \mathrm{~m}$ do solo (DAP) $\geq 45 \mathrm{~cm}$, sem nenhuma intervenção posterior; $\mathrm{T}_{2}, \mathrm{~T}_{3}$ e $\mathrm{T}_{4}$ : exploração de árvores comerciais com DAP $\geq 55 \mathrm{~cm}+$ desbaste de espécies não comerciais com DAP a partir de $45 \mathrm{~cm}$, para reduzir a área basal em $20 \%, 30 \%$ e $50 \%$ da original, respectivamente. 


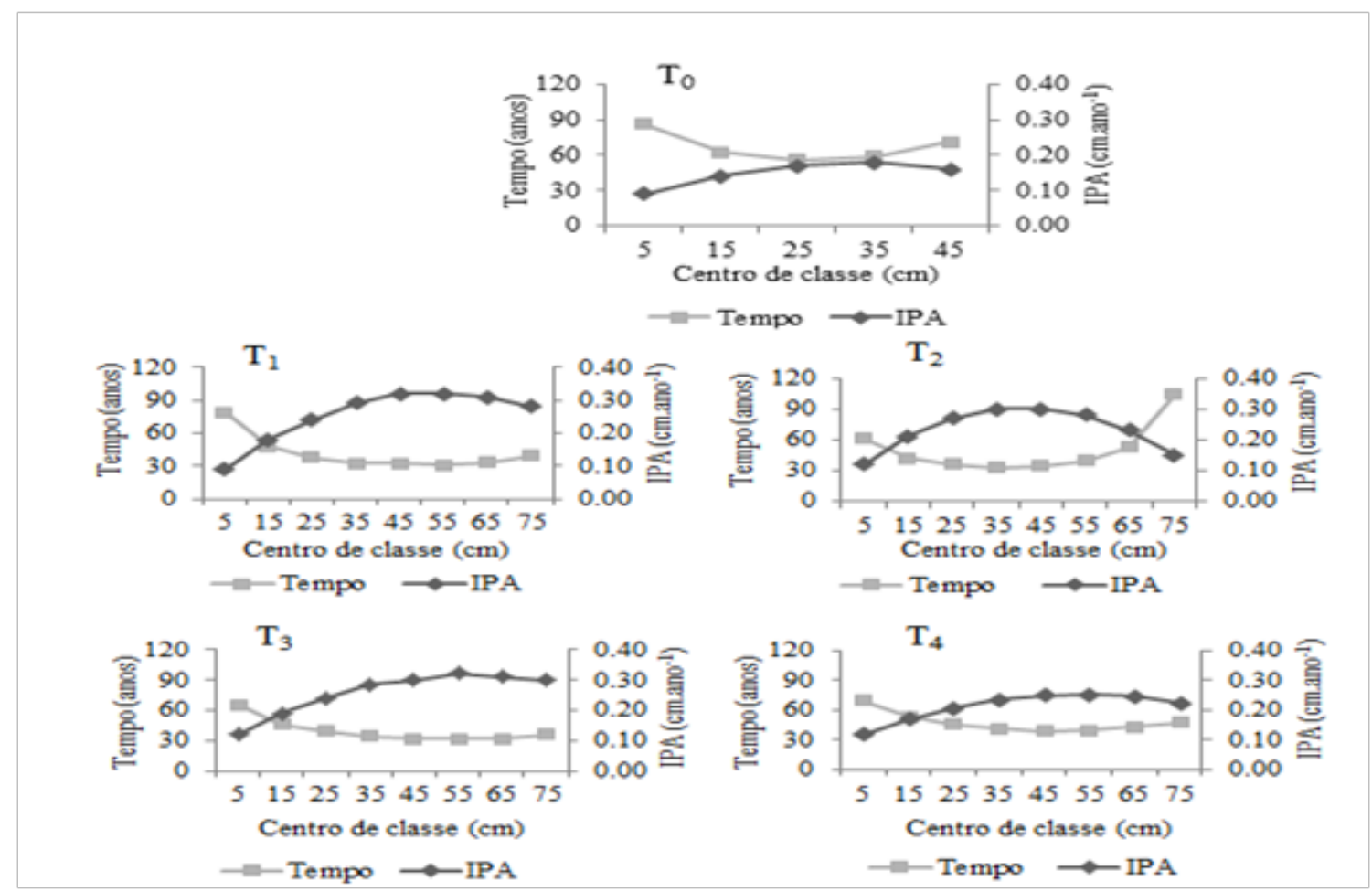

Figura 3. Tempo de passagem (anos) e incremento periódico anual médio (IPA) em diâmetro por classe de diâmetro (DAP) de Minquartia guianensis, na Floresta Nacional do Tapajós, Belterra, PA. $\mathrm{T}_{0}$ : tratamento controle, floresta sem intervenção; $\mathrm{T}_{1}$ : exploração de espécies comerciais madeireiras com diâmetro a 1,30 m do solo (DAP) $\geq 45 \mathrm{~cm}$, sem nenhuma intervenção posterior; $\mathrm{T}_{2} \mathrm{~T}_{3} \mathrm{e} \mathrm{T}_{4}$ : exploração de árvores comerciais com $\mathrm{DAP} \geq 55 \mathrm{~cm}+$ desbaste de espécies não comerciais com DAP a partir de $45 \mathrm{~cm}$, para reduzir a área basal em $20 \%, 30 \%$ e $50 \%$ da original, respectivamente.

$\mathrm{O} \mathrm{T}_{0}$ precisará de mais 86 anos para ingressar na classe de 15-25 cm, no entanto, $\mathrm{T}_{1}, \mathrm{~T}_{2}, \mathrm{~T}_{3}$ e $\mathrm{T}_{4}$ apresentou comportamento diferente, necessitando de 79,61, 65 e 62 anos, respectivamente. De maneira geral, uma árvore de regeneração levará, em média, 318, 253, 212, 224, 215 anos para deixar a classe anterior e ingressar no estoque de árvores no centro de classe de $50 \mathrm{~cm}$ nos tratamentos $\mathrm{T}_{0}, \mathrm{~T}_{1}, \mathrm{~T}_{2}, \mathrm{~T}_{3}$ e $\mathrm{T}_{4}$, respectivamente.

O tempo necessário para as árvores da classe de diâmetro de 45-55 cm (centro de classe de $50 \mathrm{~cm}$ ) ultrapassarem a classe de $55-65 \mathrm{~cm}$ é de aproximadamente $71\left(\mathrm{~T}_{0}\right), 32\left(\mathrm{~T}_{1}\right), 34\left(\mathrm{~T}_{2}\right), 32\left(\mathrm{~T}_{3}\right)$ e $34 \operatorname{anos}\left(\mathrm{T}_{4}\right)$. Dessa forma, decorrido esse período para cada tratamento, todas as árvores da classe diamétrica de $45-55 \mathrm{~cm}$ já terão ultrapassado o diâmetro mínimo de corte (DMC) de $50 \mathrm{~cm}$.

Por outro lado, quando se analisa as classes de tamanho com DAP $\leq 50 \mathrm{~cm}$, as árvores concentradas na classe de $35-45 \mathrm{~cm}$ apresentam ciclo de corte dentro do que é estabelecido na legislação, que considera para o manejo sustentável um ciclo de no mínimo 25 anos e no máximo 35 anos (Conselho Nacional do Meio Ambiente,
2009). Dessa forma, todos os tratamentos avaliados, com exceção de $\mathrm{T}_{0}$, estão dentro desse intervalo, pois na classe de $35-45 \mathrm{~cm}$ apresentaram $32\left(\mathrm{~T}_{1}\right), 33\left(\mathrm{~T}_{2}\right), 34$ $\left(\mathrm{T}_{3}\right)$ e $34\left(\mathrm{~T}_{4}\right)$ anos.

$\mathrm{O}$ tratamento $\mathrm{T}_{2}$ apresentou maior tempo de passagem na última classe de diâmetro. M. guianensis, ao atingir $50 \mathrm{~cm}$, que é o diâmetro mínimo de corte estabelecido na legislação, apresenta lentidão ou estagnação nos processos de dinâmica de crescimento com o decorrer dos anos, podendo gerar desperdício de madeira. Dessa forma, pode-se considerar como diâmetro ideal de corte árvores com DAP entre 35 e $45 \mathrm{~cm}$, em razão do melhor ritmo de crescimento obtido, menor tempo de passagem entre as classes diamétricas e as dimensões adequadas para uso (Souza et al., 2005).

Segundo Souza et al. (2005), indivíduos adultos podem ser importantes fontes de sementes nas áreas manejadas. Portanto, o corte de árvores com DAP $\leq$ $50 \mathrm{~cm}$ deve ser feito de forma criteriosa e seletiva, minimizando os efeitos do manejo e proporcionando o recrutamento contínuo de indivíduos para as classes diamétricas maiores. 
Tabela 4. Idade relativa, incremento periódico anual (IPA) e tempo de passagem, por classe de diâmetro de Minquartia guianensis, na Floresta Nacional do Tapajós, Belterra, PA.

\begin{tabular}{|c|c|c|c|c|c|c|}
\hline Tratamentos & $\begin{array}{c}\text { Centro de classe } \\
\text { (cm) }\end{array}$ & $\begin{array}{l}\text { IPA observado } \\
\left(\mathrm{cm} \text { ano }^{-1}\right)\end{array}$ & $\begin{array}{l}\text { IPA estimado } \\
\left(\mathbf{c m} \text { ano }^{-1}\right)\end{array}$ & $\begin{array}{l}\text { Idade mínima } \\
\quad \text { (anos) }\end{array}$ & $\begin{array}{l}\text { Idade } \\
\text { máxima } \\
\text { (anos) }\end{array}$ & $\begin{array}{c}\text { Tempo de } \\
\text { passagem } \\
\text { (anos) }\end{array}$ \\
\hline \multirow{5}{*}{$\mathrm{T}_{0}$} & $5-15$ & 0,110 & 0,091 & 55 & 140 & 86 \\
\hline & $15-25$ & 0,201 & 0,145 & 141 & 202 & 62 \\
\hline & $25-35$ & 0,143 & 0,174 & 203 & 258 & 56 \\
\hline & $35-45$ & 0,120 & 0,179 & 259 & 317 & 59 \\
\hline & $45-55$ & 0,176 & 0,159 & 318 & 389 & 71 \\
\hline \multirow{7}{*}{$\mathrm{T}_{1}$} & $5-15$ & 0,124 & 0,089 & 56 & 134 & 79 \\
\hline & $15-25$ & 0,140 & 0,176 & 135 & 182 & 48 \\
\hline & $25-35$ & 0,325 & 0,242 & 183 & 220 & 38 \\
\hline & $35-45$ & 0,315 & 0,290 & 221 & 252 & 32 \\
\hline & $45-55$ & 0,338 & 0,317 & 253 & 284 & 32 \\
\hline & $55-65$ & 0,335 & 0,325 & 285 & 315 & 31 \\
\hline & $65-75$ & 0,243 & 0,313 & 316 & 348 & 33 \\
\hline \multirow{7}{*}{$\mathrm{T}_{2}$} & $5-15$ & 0,114 & 0,125 & 40 & 100 & 61 \\
\hline & $15-25$ & 0,317 & 0,209 & 101 & 142 & 42 \\
\hline & $25-35$ & 0,272 & 0,266 & 143 & 178 & 36 \\
\hline & $35-45$ & 0,278 & 0,297 & 179 & 211 & 33 \\
\hline & $45-55$ & 0,297 & 0,300 & 212 & 245 & 34 \\
\hline & $55-65$ & 0,230 & 0,278 & 246 & 284 & 39 \\
\hline & $65-75$ & 0,234 & 0,228 & 285 & 377 & 53 \\
\hline \multirow{7}{*}{$\mathrm{T}_{3}$} & $5-15$ & 0,118 & 0,124 & 40 & 104 & 65 \\
\hline & $15-25$ & 0,270 & 0,191 & 105 & 150 & 46 \\
\hline & $25-35$ & 0,201 & 0,242 & 151 & 189 & 39 \\
\hline & $35-45$ & 0,247 & 0,281 & 190 & 223 & 34 \\
\hline & $45-55$ & 0,316 & 0,305 & 224 & 255 & 32 \\
\hline & $55-65$ & 0,371 & 0,316 & 256 & 287 & 32 \\
\hline & $65-75$ & 0,326 & 0,313 & 288 & 319 & 32 \\
\hline \multirow{7}{*}{$\mathrm{T}_{4}$} & $5-15$ & 0,149 & 0,123 & 41 & 102 & 62 \\
\hline & $15-25$ & 0,249 & 0,207 & 103 & 145 & 43 \\
\hline & $25-35$ & 0,279 & 0,264 & 146 & 180 & 35 \\
\hline & $35-45$ & 0,270 & 0,294 & 181 & 214 & 34 \\
\hline & $45-55$ & 0,303 & 0,299 & 215 & 248 & 34 \\
\hline & $55-65$ & 0,333 & 0,277 & 249 & 287 & 39 \\
\hline & $65-75$ & 0,142 & 0,230 & 288 & 339 & 52 \\
\hline
\end{tabular}

T0: tratamento controle, floresta sem intervenção; T1: Exploração de espécies comerciais madeireiras com diâmetro a 1,30 m do solo (DAP) $\geq 45 \mathrm{~cm}$, sem nenhuma intervenção posterior; T2, T3 e T4: Exploração de árvores comerciais com DAP $\geq 55 \mathrm{~cm}+$ desbaste de espécies não comerciais com DAP a partir de $45 \mathrm{~cm}$, para reduzir a área basal em $20 \%, 30 \%$ e $50 \%$ da original, respectivamente.

Informações obtidas com o monitoramento de parcelas permanentes podem ser úteis ao planejamento do manejo florestal, como demonstrado por Sist et al. (2014), ao recomendarem que árvores com DAP $\geq 110$ $\mathrm{cm}$ não poderiam ser derrubadas, considerando que grandes árvores são importantes para a manutenção do equilíbrio local no balanço de carbono. Da mesma forma, os resultados demonstram que não há justificativas ecológicas e, provavelmente, nem comerciais para a derrubada de árvores de M. guianensis com DAP $\geq 50$, $\mathrm{cm}$ como preconizado pela legislação ambiental vigente. 


\section{Conclusões}

Em todos os tratamentos avaliados ocorreu a diminuição do crescimento diamétrico a partir do $\mathrm{DAP} \geq 50 \mathrm{~cm}$, resultado que indica incoerência entre o parâmetro legal de DAP mínimo de corte estabelecido pela legislação ambiental brasileira e as características ecológicas da Minquartia guianensis.

Recomenda-se a manutenção das árvores de $M$. guianensis com DAP $\geq 50 \mathrm{~cm}$, para a produção de sementes e como estoque genético local. As classes inferiores $(35-45 \mathrm{~cm})$ que apresentaram maior produtividade e menor tempo de passagem, com características comerciais desejáveis e que compõem o estoque ativo comercialmente, poderiam ser manejadas.

\section{Agradecimentos}

À Embrapa Amazônia Oriental, por disponibilizar os dados e incentivar a pesquisa; e ao Conselho Nacional de Desenvolvimento Científico e Tecnológico (CNPq), pela concessão da bolsa de estudos à primeira autora.

\section{Referências}

Azevedo, C. P. et al. Efeito da exploração de madeira e dos tratamentos silviculturais no agrupamento ecológico de espécies. Floresta, v. 38, n. 1, p. 53-69, 2008. DOI: 10.5380/rf.v38i1.11027.

Azevedo, C. P. et al. Eficiência de tratamentos silviculturais por anelamento na Floresta do Jari, Amapá. Floresta, v. 42, n. 2, p. 315324, 2012. DOI: 10-.5380/rf.v42i2.17610.

Braz, E. M. et al. Criteria to be considered to achieve a sustainable second cycle in Amazon Forest. Pesquisa Florestal Brasileira, v. 35, n. 83, p. 209-255, 2015. DOI: 0.4336/2015.pfb.35.83.941.

Braz, E. M. et al. Manejo da estrutura diamétrica remanescente de florestas tropicais. Ciência Florestal, v. 22, n. 4, p. 787-794, 2012. DOI: $10.5902 / 198050987559$.

Camargo, J. L. C. \& Ferraz, I. D. K. Acariquara-roxa, Minquartia guianensis Aubl. Informativo Técnico Rede de Sementes da Amazônia, n. 10, 2005.

Cardoso Junior, R. C. et al. Tratamento silvicultural e incremento diamétrico de Plantonia insignis Mart. (Clusiaceae) "bacurizeiro" em duas florestas secundárias em Bragança, PA, Brasil. Revista Árvore, v. 38 , n. 5, p. 889-898, 2014. DOI: 10.1590/S010067622014000500013.

Carvalho, J. O. P. Changes in the spatial distribution of tree species in a terra firme rain forest in Brazilian Amazonia after logging. Revista de Ciências Agrárias, n. 37, p. 53-70, 2002.
Conselho Nacional do Meio Ambiente (Brasil). Resolução $\mathbf{n}^{\mathbf{0}}$ 406, de 06 de fevereiro de 2009. Estabelece os parâmetros técnicos a serem adotados na elaboração, apresentação, avaliação técnica e execução de Plano de Manejo Florestal Sustentável - PMFS com fins madeireiros, para florestas nativas e suas formas de sucessão no bioma Amazônia. Disponível em: <http://www.mma.gov.br/port/ conama/legiabre.cfm?codlegi=597>. Acesso em: 15 maio 2016.

Costa, D. H. M. et al. Crescimento de árvores em uma área de terra firme na Floresta Nacional do Tapajós após a colheita de madeira. Revista Ciências Agrárias, n. 50, p. 63-76, 2008.

Cunha, T. A. \& Finger, C. A. G. Competição assimétrica e o incremento diamétrico de árvores individuais de Cedrela odorata L. na Amazônia Ocidental. Acta Amazonica, v. 43, n. 1, p. 9-18, 2013. DOI: $10.1590 / \mathrm{S} 0044-59672013000100002$.

Espírito-Santo, F. D. B. et al. Análise da composição florística e fitossociológica da Floresta Nacional do Tapajós com apoio geográfico de imagens de satélites. Acta Amazonica, v. 35, n. 2, p. 155-173, 2005. DOI: 10.1590/S0044-59672005000200006.

Hirai, E. H. et al. Efeito da exploração florestal de impacto reduzido sobre a regeneração natural em uma floresta densa de terra firme no município de Paragominas na Amazônia brasileira. Scientia Forestalis, v. 40, n. 95, p. 306-315, 2012.

IBGE. Manual técnico da vegetação brasileira. Rio de Janeiro, 2012. 271 p.

Instituto Nacional de Meteorologia (Brasil). Estação meteorológica de observação de superfície convencional. Brasília, DF, 2016. Banco de dados. Disponível em: <http://www.inmet.gov.br/sim/ sonabra/convencionais.php>. Acesso em: 15 jan. 2016.

Marenco, R. A. \& Vieira, G. Specific leaf area and photosynthetic parameters of tree species in the forest understorey as a function of the microsite light environment in Central Amazonia. Journal of Tropical Forest Science, v. 17, p. 265-278, 2005.

Nebel, G. Minquartia guianensis Aubl.: uso, ecologia y manejo em forestería y agroforestería. Folia Amazónica, v. 10, n. 1-2, p. $1-23,2000$.

Oliveira, L. C. et al. Efeito da exploração de madeira e tratamentos silviculturais sobre a estrutura horizontal de uma área de 136 ha na Floresta Nacional do Tapajós, Belterra-Pará. Revista de Ciências Agrárias, v. 46, p. 195-214, 2006.

Reis, L. P. et al. Crescimento de mudas de Parkia gigantocarpa Ducke, em um sistema de enriquecimento em clareiras após a colheita de madeira. Ciência Florestal, v. 24, n. 2, p. 431-436, 2014. DOI: 10.5902/1980509814583.

Silva, J. N. M. et al. (Ed.). Diretrizes para a instalação e medição de parcelas permanentes em florestas naturais da Amazônia Brasileira. Belém, PA: Embrapa, 2005. 36 p.

Silva, J. N. M. et al. Growth and yield of a tropical rain forest in the brazilian Amazon 13 years after logging. Forest Ecology and management, v. 71 , n. 3, p. 267-274, 1995. DOI: 10.1016/03781127(94)06106-S. 
Silva Jardim, F. C. S. \& Soares, M. S. Comportamento de Sterculia pruriensis (Aubl.) Schum. em floresta tropical manejada em MojuPará. Acta Amazonica, v. 40, n. 3, p. 535-542, 2010. DOI: 10.1590/ S0044-59672010000300012.

Sist, P. et al. Large trees as key elements of carbono storage and dynamics after selective logging in the Eastern Amazon. Forest Ecology and Management, v. 38, p. 103-109, 2014. DOI: 10.1016/j. foreco.2014.01.005.

Soares, M. S. et al. Avaliação do crescimento diamétrico de Sterculia pruriensis (Aublet) Schumann - Sterculiaceae (axixá), em clareiras da floresta tropical explorada seletivamente, em Moju-Pará. Revista Brasileira de Biociências, v. 5, n. 1, p. 699-701, 2010.

Souza, A. C. B. et al. Estabelecimento de plântulas de Minquartia guianensis (Olacaceae) em uma área submetida ao corte seletivo na Amazônia Central. In: Ecologia da Floresta Amazônica 2005. Manaus: INPA, 2005. Projeto Dinâmica biológica de fragmentos Florestais. Disponível em: < http://pdbff.inpa.gov.br/cursos/efa/ livro/2005/pdfs/po2g2.pdf>. Acesso em: 15 jan. 2016.
Souza, A. L. \& Soares, C. P. B. Florestas nativas: estrutura, dinâmica e manejo. Viçosa, MG: Ed. da UFV, 2013. 322 p.

Souza, D. V. et al. Crescimento de espécies arbóreas em uma floresta natural de terra firme após a colheita de madeira e tratamentos silviculturais, no município de Paragominas, Pará, Brasil. Ciência Florestal, v. 25, n. 4, p. 873-883, 2015. DOI: $10.5902 / 1980509820585$

Souza, P. A. et al. Idade relativa como subsídio à determinação de ciclo de corte no manejo sustentável de povoamentos florestais nativos. Revista Árvore, v. 17, n. 1, p. 100-116, 1993.

Taffarel, M. et al. Efeito da silvicultura pós-colheita na população de Chrysophyllum lucentifolium Cronquist (Goiabão) em uma floresta de terra firme na Amazônia brasileira. Revista Árvore, v. 38, p. 1045 1054, 2014. DOI: 10.1590/S0100-67622014000600009. 
